LAWRENCE LIVERMORE N A TION A L LABORATORY

\title{
Managing Complex and Dynamic Systems For The Future
}

E. J. Jones

November 14, 2003

1st International Symposium on Systems \& Human Science for Safety, Security and Dependability

Osaka, Japan

November 18, 2003 through November 20, 2003 
This document was prepared as an account of work sponsored by an agency of the United States Government. Neither the United States Government nor the University of California nor any of their employees, makes any warranty, express or implied, or assumes any legal liability or responsibility for the accuracy, completeness, or usefulness of any information, apparatus, product, or process disclosed, or represents that its use would not infringe privately owned rights. Reference herein to any specific commercial product, process, or service by trade name, trademark, manufacturer, or otherwise, does not necessarily constitute or imply its endorsement, recommendation, or favoring by the United States Government or the University of California. The views and opinions of authors expressed herein do not necessarily state or reflect those of the United States Government or the University of California, and shall not be used for advertising or product endorsement purposes. 


\title{
Managing Complex and Dynamic Systems for the Future
}

\author{
E. D. Jones \\ Lawrence Livermore National Laboratory \\ 7000 East Avenue, L-634 \\ Livermore, California 94551, USA \\ jones37@llnl.gov
}

\begin{abstract}
The challenges of modern complicated systems regarding their design, analysis, and management are put in a historical context to better propose a framework for the future involving complementary uses of testing, modeling, and performance functions.
\end{abstract}

Keywords: complex systems, nonlinear dynamics, uncertainty, performance.

\section{Introduction}

As scientists and engineers involved with complex and dynamic systems, we are at an important point in intellectual history, and we thus have a special responsibility to society. The unusual behavior of complicated engineered systems - with their attendant potential benefits and dangers - have been foreshadowed and anticipated for some time. Examples include solitary wave phenomena (e.g., Kortwieg de Vries and sine-Gordon models); hyper-sensitivity to initial conditions (e.g., weather models); nonlinear behavior of many-body simulations (e.g., Monte Carlo and cellular automata); and, more recently, system approaches to biological and earth sciences.

Closer to the public, over time we have built systems, such as energy, information, financial, communication, and physical infrastructure networks, with relatively simple component behaviors which in connection and interdependence with other elements have become complex systems capable of exhibiting unexpected phenomena and vulnerabilities to perturbations. A recent example is the failure of the Northeast American power grid in mid-August, 2003, followed a couple of weeks later by the blackout of Italy. These power management systems were developed with hardware, software, and human operator components each well-understood using traditional engineering practices. But their collective behavior and failure, in the American instance cited, are not understood to date despite the focused efforts of the best experts in the field. Failures of complex systems on which society has come to depend may be the difference between light and dark, or even life and death.

However, we don't need just large, extensive network systems to appreciate the problems and challenges we will face in this century regarding the design, analysis, and management of complex and dynamic systems. Smaller, more focused, semi-automatic or robotic systems also present laboratories for the investigation of complicated processes which typically involve a mixture of machine, software, and human factors. I will use my experience with the risk analysis of a semi-robotic medical radiation treatment device, the Gamma Knife, to illustrate the potential roles of measurement, simulation, and performance objectives in managing complex and dynamic systems in the future.

We now must rapidly learn to deal with such complicated systems, because they are becoming prevalent in our societies and present practical problems. Happily, we have available techniques and tools, especially computational capabilities, to aid us. We also need paradigms or frameworks for thinking anew about systems in an increasingly nonlinear world. This paper suggests a path forward based on complementary uses of system measurement, simulation, and performance functions.

\section{Systems Analysis and Measurement}


In order to foresee complex systems analysis, it is useful to put our predicament in an historical perspective. Since the beginning of the Age of Reason, Western thinkers have struggled with the respective roles and dilemmas associated with determinism and uncertainty. By the early 1800's, a synthesis of these ideas was obtained, as embodied in the work of Laplace. $^{1,2}$ Laplace's astronomical work was a completely mechanical explanation of the solar system based on Newtonian principles, i.e., it was the deterministic 'celestial mechanics'. However, astronomical measurements have discrepancies or errors among them, and it is problematic to know which result is the usable one. The solution to this problem was the 'law of errors' (i.e., errors are normally distributed such that the deviation from the mean is as predictable as the mean itself as a function of the number of observations) which arose out of two closely related ideas: probability theory, which sought to understand chance events, and statistics which sought to measure fluctuating phenomena. Laplace was explicit about the probabilistic nature of statistical calculations: we can never know with absolute certainty; we can only know with greater or lesser degrees of probability. With the 'law of errors', statistics and probability theory allowed scientists to achieve far greater degrees of precision then they had imagined possible.

In general, the concepts of that era have propagated forth to inform systems thinking today. They are embedded in powerful conventional constructs of analysis: linear causality, precision certainty, reversibility, reductionism, and induction/deduction. Unfortunately, some or all of these concepts may not apply to evaluations of the complicated phenomena of modern complex and dynamic systems.

For instance, statistics can conquer uncertainty, but statistics needs proper measurements or tests to be made. Proper measurement involves the assumption that a piece of the system can be partitioned and isolated to be measured; the component measurement is independent of the other parts of the system; and the results can be placed back in the aggregated system without prejudice. This is a lot to take for granted with modern systems, which typically contain hardware, software, and human elements coupled together. To illustrate this, Fig. 1 summarizes the nature of errors or failures for such components.

\begin{tabular}{|l|c|c|}
\hline & Deterministic & Stochastic \\
\hline Hardware & No & Yes \\
\hline Software & Yes & No \\
\hline $\begin{array}{l}\text { Human } \\
\text { (unintentional) }\end{array}$ & Yes & Yes \\
\hline $\begin{array}{l}\text { Human } \\
\text { (intentional) }\end{array}$ & No & No \\
\hline
\end{tabular}

Fig. 1. Nature of errors or failures associated with system components.

We are familiar with hardware or machine failures, where the failures are random but their rates can be determined through statistically rigorous testing regimes. All software errors are pre-determined - they are encoded - but their occurrence depends on a conspiracy of circumstance which may not be anticipated by stochastic sampling. Unintentional human errors occur for both mechanistic and random reasons and can, in principle, have rates established through statistical tests; but all relevant conditions or environments may not be available. Intentional human acts, such as criminal or terrorist acts, are neither deterministic nor random and hence beg the use of probability. These error problems are compounded when different components interact in unexpected ways. So, in a complicated system the value of measurement is conditional.

We had to deal with such problems for a risk-based evaluation of the Gamma Knife semi-robotic medical device. ${ }^{3}$ Decomposing or partitioning of the system process into steps was aided by the linear process procedure from lesion identification, treatment planning, pre-treatment setup, to treatment. But each step contained a mix of hardware, software, and human activities and potential failure modes for which very little information was available (the Gamma Knife was a new device that had been used for a limited number of treatments and had few failures). Statistical samples with confidence levels for failures during each step were out of the question, and we thus used qualitative probability distributions constructed from sparse manufacturer, operational, anecdotal, and expert information. The data were compared in a risk profiling 
method that allowed us to compare the relative risk (based on mean values) of each process step, independent of the other steps. This provided a first order view of where important risk may lie in the performance of the system.

The bottom line of this discussion is that reductions for measurement or testing of a complex system are not adequate: they only provide an incomplete representation of the system's real state-of-affairs. Other aspects of system analysis are needed.

\section{System Simulations}

Another way to represent the behavior of a system is through modeling or simulation. This, of course, requires another type of abstraction, and partial view, of the complex system involving a choice of mechanisms to model and preferences for the generation of certain types of system data or behaviors. But the coordination of the nature of the simulations with the system features testing and measurement capabilities can lead to a more faithful representation of the system as a whole. Computer simulations are a powerful way of gaining insight into complex system processes and are substantially aided by modern developments in high-performance computing, visualization tools, and the rise of interdisciplinary research.

System simulations can be profitably used in different ways. One approach is to look at many solutions at once to develop a sophisticated understanding of the global structure of an ensemble of system solutions with respect to its underlying mechanisms. This may entail looking for specific patterns in the system performance data; or using the 'geometry' of the solutions to guide optimal performance strategies against several competing constraints. This approach is used in planning spacecraft trajectories in celestial mechanics; and in some forms of weather prediction. Another application is sensitivity studies where fluctuations or perturbations are introduced to study oscillations or limit cycles of the system, from which it is possible to design strategies that ensure stable operation. Example arenas are manufacturing processes and transportation networks. The computer modeling may be direct, in the sense of starting from basic mechanisms to develop system states-of-affairs or outcomes. On the other hand, as demonstrated by Lawrence Livermore fusion scientists, codes can be developed where the performance outcomes are provided as inputs, and the optimal system design to meet those requirements is generated.

In the case of the Gamma Knife study, sequential Monte Carlo simulations were employed to generate a multitude of treatment scenarios in order to explore the risk space of the system. The system process steps each had qualitative (using relative scales) probability distributions for both equipment and human errors or failures and also distributions for the magnitudes of the errors. A Monte Carlo sampling technique was applied to each step distribution to determine if an error occurred in that step, and if so, what was its sampled magnitude; and then the errors were aggregated probabilistically using the logical relations among the system components. Thus, for each scenario the aggregate likelihood of error and its magnitude (i.e., its consequence) was recorded along with the risk contributors. Through 100,000 such simulations to reach convergence, a picture of the distribution of risks emerged along with the identification of the most significant contributors to the highest risks. The results of these simulations revealed different significant risk contributors than the relative point risk estimates mentioned in the previous section. This was due to the unexpected conspiracy effects of the interactions among process events, especially with respect to the tails of their distributions. In other word, the shape of the distributions turned out to be more important then their mean values, through nonlinear interactions which were only realized upon simulation. These effects were later validated by real events.

As mentioned above, the important science and art of complex systems analysis is to coordinate both system testing and simulation to achieve a more robust understanding and management of the system. Simulation can help direct what system features should be measured and to what extent; and, of course, system testing/measurement will inform the desired characteristics of the simulation model. The desirable goal is to achieve a high level of correlation among measurements, simulations, and phenomenological performance figures-of-merit. For complex systems, we probably cannot disentangle the nature of the correlations between deterministic and stochastic effects; but having confidence in the correlations 
themselves is extremely valuable regardless of their pedigree.

\section{Bounding System Performance}

The third complementary aspect, in addition to measurement and simulation, needed for complex system evaluation and management is realistic performance bounds and associated figures-of-merit for system performance. Dynamic systems become problems when certain behaviors become unbounded or far exceed expected limits, and these can be facilitated if appropriate performance objectives are not employed and integrated with the design or analysis from the beginning. As an analogy, if nutrients and space are unlimited, bacteria will divide steadily and increase exponentially. But if resources are limited, the bacteria proliferation rate will drop or the death rate will increase, and the population will stabilize. Similarly, the scientist and engineer need to establish and impose limits for their systems, to ensure that unwanted system excursions do not occur. These can be used to coordinate the measurement and simulation activities for system analysis, so that the correlated representation of the system maps into performance figures-of-merit, and vice versa, to aid system control and management.

One useful approach to bounding system behavior is to delimit the risks, between residual risk of little or no interest and unacceptable risk; and to delimit performance, for example, between desirable performance objectives, such as throughput or maintainability, and available resources or options (money, equipment, people, etc.) which are always limited. One way to represent these bounds is in a risk-based framework as illustrated in Fig. 2.

The aim of the establishment of risk goals is to define the regions of prudent or acceptable risks, thus allowing systems to operate within them to maximize their benefits or utilities as resources and performance characteristics will permit. These risk goals should have both consequence and likelihood dimensions. Two limits are needed for the likelihood bounds: a 'screening' frequency below which the unwanted high consequence incident likelihood is considered negligible; and a 'target' frequency above which events for lower levels of consequence can not be allowed to occur. Thus, for each risk goal the states of the system are managed (by the available options) such that the likelihood for any consequence is between the screening and target limits.

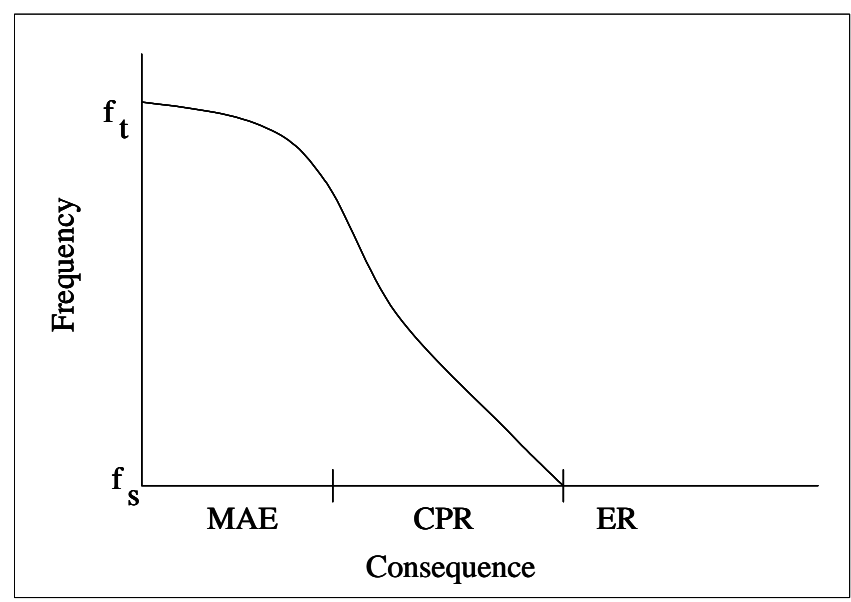

Fig. 2. Risk space defined and partitioned among screening $\left(f_{s}\right)$ and target $\left(f_{t}\right)$ frequencies and regions of Mutually Acceptable Equilibrium (MAE), Conservatively Permissible Risk (CPR), and Emergency Response (ER).

The region of 'mutually acceptable equilibrium' (MAE) refers to common system scenarios and events that occur at frequencies high enough to make them statistically observable, but the consequences tend to be small. The statistics for MAE events allow a management balance which is continuously fine-tuned and has 'preventive' qualities. Scenarios from this region might escalate into the region of 'conservatively permissible risk' (CPR) where scenarios and events are highly improbable (beyond the design basis) but the consequences may be severe. Such system endpoints need to be examined to determine what design mechanisms or barriers are required so that their likelihood is limited. For CPR scenarios, if they occur, there needs to be mitigative management options. Emergency response is invoked if mitigation fails. The sequence of preventive, mitigative, and emergency response management constitute a defense-in-depth against runaway system behavior. Of course, one always wants to move the risks, by cost effective system design changes or management practices, from higher to lower risk regions.

The risk goals, management options, and other objectives and constraints bound the complex system evaluation problem. They provide the endpoints of 
interest to scenarios and serve to reach problem closure by providing a quantitative feel for the magnitude of the risks relative to the various management strategies.

In the case of the Gamma Knife study, system component measurements were used in Monte Carlo simulations of a multitude of scenarios to delineate the risk space for the Gamma Knife system. The results indicated a relatively large population of scenarios in high risk regions, which exceeded desirable risk goals. Inspection of these scenarios identified certain process tasks that are significant risk contributors. By making changes in procedures and design features associated with these tasks, subsequent simulations indicated a substantial reduction in potential high risk scenarios. In terms of the discussion above, these methods exemplified how scenarios in the CPR regime could be moved to the MAE regime through system feature changes.

\section{Summary}

We are faced with managing emerging complex and dynamic systems in our societies, which we depend on for our well-being, safety, and security. Their mixed attributes of mechanics, software, and human factors give rise to complications and issues heretofore unaddressed in the history of system analysis. We may not be able to depend on traditional and reliable concepts such as reductionism or induction or even direct causality, and yet we must proceed.

This paper has suggested that a science-based risk approach framework for analyzing, designing, and managing our complex systems entails a triad of complementary functions: measurement or testing, modeling or simulation, and risk-based objectives to bound performance. Probabilistic techniques play a fundamental role in combining and mapping information among these functions. Each function is inadequate in itself; but they can work together, synergistically, to help understand complex system behavior. This has been demonstrated in the case of the semi-robotic medical treatment device, the Gamma Knife. While the applications of these methods are contingent on the particular system of interest; the collection of principles they represent may be more universally applicable.

\section{References}

1) I. Hacking, The Emergence of Probability: A Philosophical Study of Early Ideas about Probability, Induction, and Statistical Inference, Cambridge University Press, 1975.

2) P.L. Bernstein, Against the Gods; The Remarkable Story of Risk, John Wiley and Sons, 1996.

3) E.D. Jones, W.W. Banks, T.J. Altenbach, and L.E. Fischer, Relative Risk Analysis in Regulating the Use of Radiation-Emitting Medical Devices; A Preliminary Application, U.S. Nuclear Regulatory Commission, NUREG/CR-6323, 1995. 